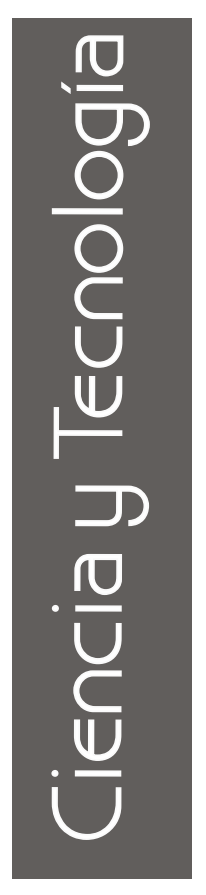

\title{
DISEÑO Y CONSTRUCCIÓN DE UNA MÁQUINA DE MOVIMIENTO PASIVO CONTINUO PARA LA TERAPIA DE RODILLA
}

\author{
Esteban Emilio Rosero G. * \\ Rodrigo Martínez D.** \\ Elmer Galvis L. ***
}

* Ingeniero Mecánico - Universidad del Valle

** M.Sc. en Automática - Profesor Asociado de la Facultad de Ingeniería - Universidad del Valle

*** M.Sc. en Ingeniería Mecánica - Profesor Asistente de la Facultad de Ingeniería - Universidad del Valle.

\section{RESUMEN}

En este artículo se presenta el diseño sistemático de un prototipo para la terapia de rodilla mediante la aplicación de conceptos integrados de diferentes áreas de la ingeniería como la automática, la mecánica, la electrónica y la medicina. Este diseño provee de una solución económica para realizar sesiones de terapia en la rodilla.

En el proceso de diseño se utilizó el Dibujo Asistido por Computadora (CAD) y la Simulación Dinámica (DS), las cuales facilitaron la construcción del prototipo. Se obtuvo un modelo analítico y se compararon con los resultados de la simulación dinámica para validar los modelos 
computacionales.

Finalmente se diseñó y construyó un prototipo así como su sistema de potencia y de control.

Palabras Claves: Movimiento Pasivo Continuo (MPC), terapia de rodilla, simulación dinámica, sistemas de control, diseño.

\section{ABSTRACT}

This article shows the systematic design of a prototype for the knee therapy using integrated concepts of different engineering areas like Automatics, Mechanics, Electronics and Medicine. This design provides a cheap solution to undertake knee therapies.

During the design process Computer Aided Design (CAD) and Dynamic Simulation (DS) tools were used in order to ease the prototype construction. An analytic model was obtained and it was compared with the dynamic simulation results to validate the computational models.

Finally, a prototype including its power system and control was designed and constructed.

Keywords: Continuous Passive Motion (CPM), knee therapy, dynamic simulation, control systems, design.

\section{INTRODUCCIÓN}

La recuperación de pacientes después de una cirugía que se lleva a cabo en alguna articulación muscoloesquelética, por huesos fracturados o problemas articulares, es manejada mediante terapias para mantener el arco de movilidad articular, prevenir la adhesión y rigidez articular y la atrofiación de los músculos, disminuir el período de hospitalización y rehabilitación, además de darle comodidad al paciente[1], [2], uno de los métodos utilizados es la terapia de MPC.

En Colombia aún no se cuenta con la facilidad de adquirir máquinas de MPC. El objetivo académico de este trabajo es generar investigación y desarrollo de tecnología propia que conlleven a la innovación, apropiando y adaptando tecnología de punta al medio nacional, así como sustitución de importaciones.

\section{ASPECTOS TEÓRICOS}

\subsection{Definición del Movimiento Pasivo}

El MPC es una modalidad de terapia postoperatoria, producido por algún mecanismo diferente al grupo muscular actuante sobre la articulación, el paciente no realiza esfuerzo para hacer el movimiento. La articulación se somete a un rango de movimiento preestablecido y por un período de tiempo determinado.

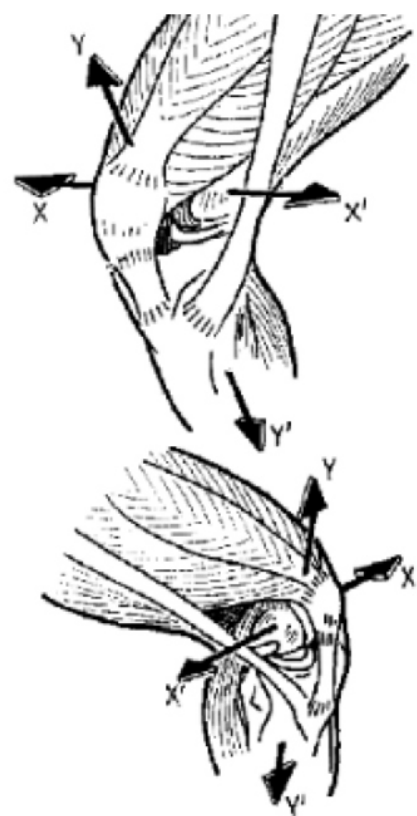

Figura 1. Movimiento de Flexión-Extensión y Ejes de Articulación

\subsection{Articulación de la Rodilla}

La rodilla es un vínculo entre la cadera y el tobillo, concilia dos imperativos contradictorios: poseer gran estabilidad y a la vez gran movilidad. Las partes importantes de la rodilla son: huesos, ligamentos, tendones y cartílagos.

\subsection{Movimientos Globales de la Rodilla}

Los principales movimientos de la rodilla [8] son (figura 1): Flexión, extensión, rotación interna y rotación externa.

\subsection{Ejes de la Articulación de la Rodilla}

El primer grado de libertad está condicionado por el eje transversal XX` (figura 1), alrededor del cual se efectúan los movimientos de flexión- 
extensión en el plano sagital [3]. El segundo grado de libertad de movimiento consiste en la rotación alrededor del eje longitudinal $Y Y^{\prime}$ de la pierna (figura 1) con la rodilla en flexión.

\section{DISEÑO E IMPLEMENTACIÓN DEL PROTOTIPO}

\subsection{Especificaciones del Diseño}

Velocidades ajustables, de 1 a 10 minutos por ciclo completo, $\left(0^{\circ}-90^{\circ}-0^{\circ}\right)$, rango de movimiento de flexión-extensión ajustables, con una amplitud de 0 a 130 grados [3], bajo peso de la estructura, ruido y vibración bajos, dentro de los rangos preestablecidos por la norma de EPA (Environmental Protection Agency), menores que $45 \mathrm{dbA}$, de fácil operación y buena confiabilidad.

El sistema de control debe cumplir con las siguientes características:

De fácil maniobrabilidad, integrado al cuerpo de la máquina de MPC, que sea liviano, que controle velocidad, posición, desplazamiento, ángulo de inicio, el tiempo necesario para cumplir todos los ciclos impuestos por el médico y que tenga bloqueo manual por dolor inesperado.

\subsection{Bases Antropométricas}

Para el diseño de la máquina se utilizan técnicas indirectas de medición antropométrica, que se basa en el uso de tablas [4], diagramas o figuras [5]. Se utilizan datos antropométricos que describen el peso promedio de los segmentos del miembro inferior, los centros de gravedad y las longitudes.

\subsection{Movimientos de la Máquina de MPC}

El movimiento de flexión-extensión es el más importante ya que abarca la mayoría de los músculos, tendones y ligamentos que se pueden atrofiar; la rotación interna-externa de alguna manera está implícita dentro del movimiento flexión-extensión.

\subsection{Proceso de Diseño de la Máquina de PMC}

El prototipo se construye usando el dibujo parametrizado por computadora CAD (Mechanical Desktop 4.0); la Ingeniería Asistida por Computadora $C A E$, se utilizó para la simulación dinámica (WORKIMG MODEL 3D) donde se obtuvo un prototipo virtual. También se desarrolló un modelo analítico. Finalmente se hizo el diseño estructural mediante el cálculo de resistencia y rigidez de los elementos más críticos así como un modelo para el sistema de control a ser implementado.

Se propone el modelo de la figura 2, este mecanismo transforma un movimiento rotacional, que es la salida del motor, en uno traslacional, que se puede ver en el movimiento del acople accionado por el tornillo de potencia.

Las cargas que están actuando en el prototipo son:

$W_{P+P}=$ Peso de la pierna y el pie

$W_{m}=$ Peso del muslo

$M=$ Momento resistivo de la articulación [6]

$\psi=$ Angulo entre el eje del muslo y el eje de la pierna.

Simulación Cinemática Analítica. Se puede calcular el recorrido $(x)$ que puede desarrollar el acople así:

$$
y=b \cos \theta+l \cos \alpha
$$

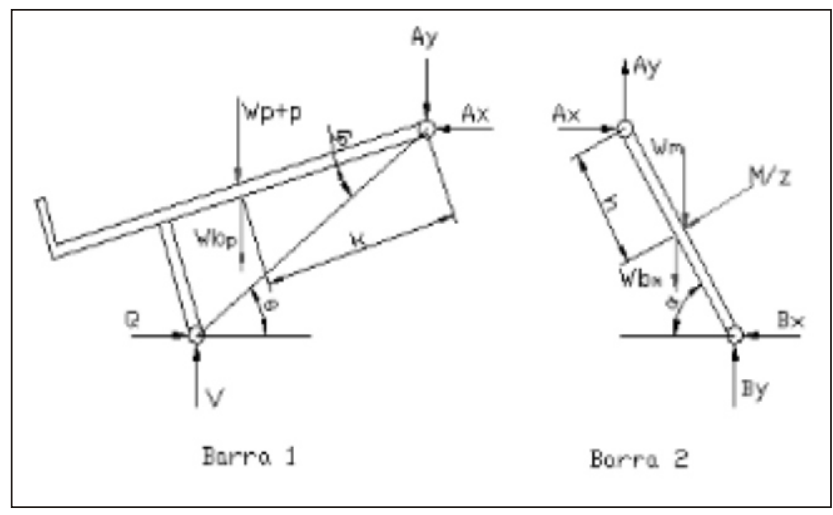

Figura 2: Variables del Prototipo para la Solución Analítica 


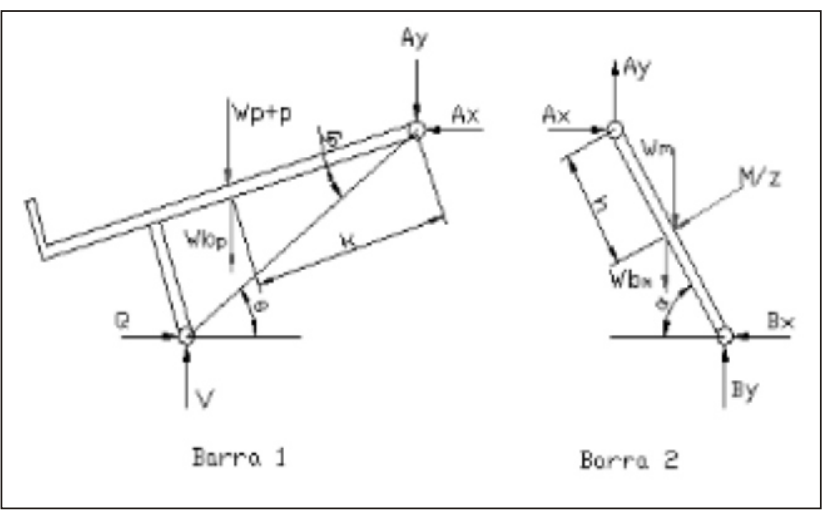

Figura 3: Diagrama de Cuerpo Libre para la Solución Analítica

El recorrido de la tuerca $x$ en función del ángulo $\psi$ formado entre la prolongación del eje del fémur y el eje de la pierna, es:

$$
x=\sqrt{b^{2}+l^{2}+2 b l \cos \left(\psi_{0}\right)}-\sqrt{b^{2}+l^{2}+2 b l \cos (\psi)}
$$

El mínimo valor del ángulo de inicio $\psi_{0}$ debe ser aproximadamente $5^{\circ}$, para que no se alineen la base de la pierna y la base del muslo.

Solución Analítica de Fuerzas. Se calcula la carga que se genera en el eje del tornillo en función del ángulo de la pierna debido a los pesos de los segmentos del miembro inferior.

Realizando el diagrama de cuerpo libre (figura 3) con las longitudes determinadas (figura 2) y aplicando las leyes de Mewton y el sistema de fuerzas equivalentes de los cuerpos rígidos se obtiene:

En la barra 1:

$$
\begin{gathered}
\Sigma F_{y}=0=V-W_{p+p}-A_{y}-W b_{p} \\
\sum F_{x}=0=Q-A_{x} \\
\sum M_{A}=0=-W_{p+p} n \cos \left(\theta-\varsigma_{0}\right) \\
-W b_{p} k \cos \left(\theta-\varsigma_{0}\right)+V b \cos \theta-Q b \operatorname{sen} \theta
\end{gathered}
$$

Donde:

$W b p=$ Peso del soporte de la pierna y el pie.

En la barra 2:

$$
\begin{aligned}
& \sum F_{y}=0=A_{y}+B_{y}-W_{m}-\frac{M}{x} \cos \alpha-W b_{m} \\
& \sum F_{x}=0=A_{x}+B_{x}-\frac{M}{x} \operatorname{sen} \alpha
\end{aligned}
$$

$$
\begin{aligned}
& \sum_{M} M_{A}=0=W_{m} z \cos \alpha+W b_{m} h \cos \alpha+(8) \\
& \frac{M}{z} z+B_{x} \operatorname{sen} \alpha-B_{y} l \cos \alpha
\end{aligned}
$$

Donde:

$$
W b_{m}=\text { Peso del soporte del muslo }
$$

Mo se consideran las aceleraciones de las barras debido a que son aproximadamente cero.

Entonces la carga de flexión $V$ (figura 4) del tornillo de potencias es:

$$
\begin{aligned}
& W_{m}\left(1-\frac{z}{l}\right)+\frac{M}{z}\left(\cos \alpha-\frac{z}{l \cos \alpha}+\frac{\operatorname{sen}^{2} \alpha}{\cos \alpha}\right) \\
& V=\frac{+W_{p+p}\left(\frac{n \cos \left(\theta-\varsigma_{0}\right)}{b \operatorname{sen} \theta} \frac{\operatorname{sen} \alpha}{\cos \alpha}+l\right)}{1+\frac{\cos \theta \operatorname{sen} \alpha}{\operatorname{sen} \theta \cos \alpha}}+ \\
& W b_{m}+\frac{W b_{m} h}{l}
\end{aligned}
$$

Que se puede expresar en función del ángulo de la rodilla $\psi$ utilizando las expresiones:

$$
\begin{gathered}
\operatorname{sen} \theta=\frac{l \operatorname{sen} \psi}{\left(b^{2}+l^{2}+2 b l \cos \psi\right)^{1 / 2}} \\
\operatorname{sen} \alpha=\frac{b \operatorname{sen} \psi}{\left(b^{2}+l^{2}+2 b l \cos \psi\right)^{1 / 2}} \\
\cos \alpha=\sqrt{1-\frac{b^{2} \operatorname{sen}^{2} \psi}{\left(b^{2}+l^{2}+2 b l \cos \psi\right)}} \\
\cos \theta=\sqrt{1-\frac{l^{2} \operatorname{sen}^{2} \psi}{\left(b^{2}+l^{2}+2 b l \cos \psi\right)}}
\end{gathered}
$$

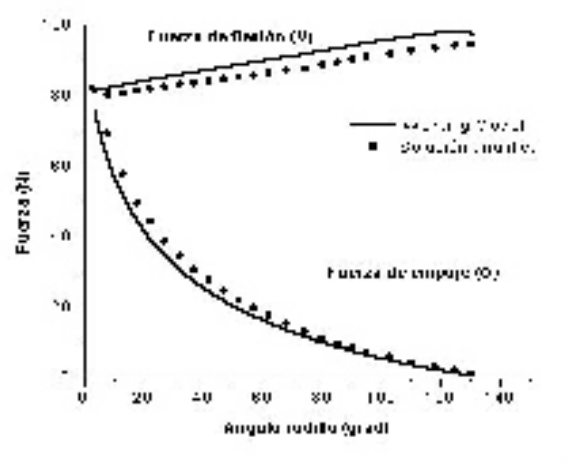

Figura 4: Comparación de las Fuerzas de Flexión y de Empuje de Working Model y de la Solución Analítica 
La carga de empuje axial Q (figura 4) que actúa en la dirección axial del tornillo es:

$$
Q=V \frac{\cos \theta}{\operatorname{sen} \theta}-\frac{\left(W_{p+p} n+W b_{p} k\right) \cos \left(\theta-\varsigma_{0}\right)}{b \operatorname{sen} \theta}
$$

Con la carga de empuje $Q$ ahora se puede obtener el torque necesario para vencer la carga (figura 5), por medio de la expresión:

$$
T_{s}=\frac{F_{x} d_{m}}{2}\left(\frac{P \cos \lambda+\pi \mu d_{m}}{\pi d_{m} \cos \lambda-\mu p}\right)
$$

Donde:

$F_{x}=$ Fuerza en la dirección axial del tornillo

$d_{m}=$ Diámetro medio del tornillo

$p=$ Paso del tornillo

$\mu=$ Coeficiente de fricción

$\lambda=$ Angulo de la rosca

$F_{x}=Q+\mu V$

y la potencia (figura 5) necesaria para el sistema se calcula a partir de la expresión:

Donde:

$$
P=\frac{2 \pi n T_{s}}{60}
$$

$P=$ Potencia

$n=r p m$

$T=$ Torque

Simulación Dinámica en Working Model. Para la generación de los movimientos de la máquina de MPC por medio de Working Model se aplicó a la tuerca una velocidad lineal igual a la generada por

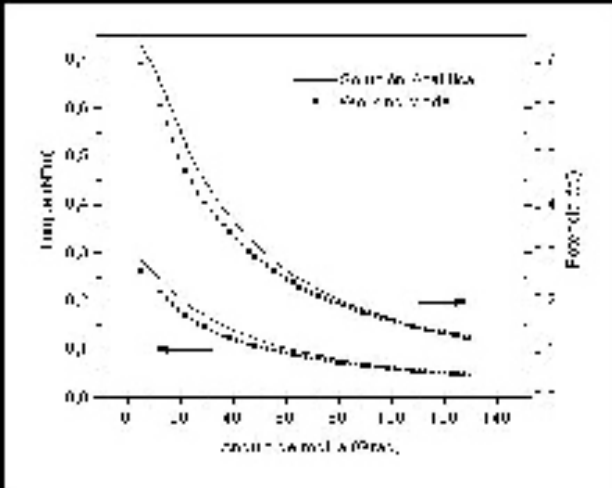

Figura 5: Comparación de Torque y Potencia de Working Model y la Solución Analítica

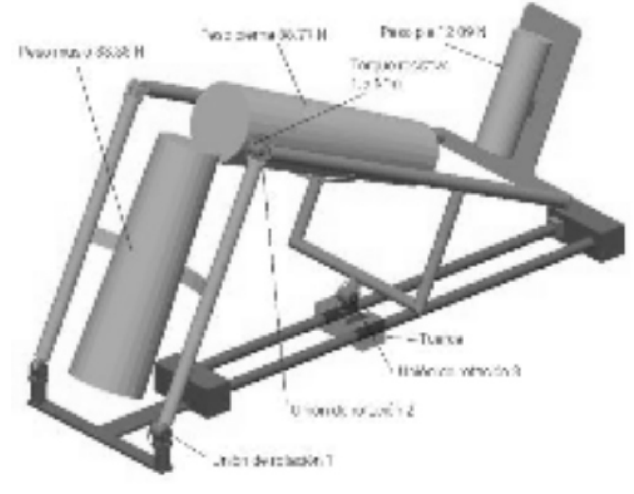

Figura 6: Modelo para la Simulación Dinámica por Working Model

el giro del tornillo. Se añade el torque resistivo de $1,5 \mathrm{~N}^{*} \mathrm{~m}$ recomendado por Hiromichi Fujie [6]. El modelo se muestra en la figura 6 . La tuerca se mueve horizontalmente paralela a los dos ejes para la máquina MPC.

Se determina la fuerza de flexión $V$ y la fuerza de empuje o axial $Q$ (figura 4). A partir de la fuerza de empuje o axial se calcula el torque y potencia (figura 5) requerida para el sistema, donde la fuerza de empuje $F_{x}=Q+\mu V$. Comparando la Simulación Dinámica con la solución analítica se puede ver que tienen comportamiento similar, difieren un poco en las magnitudes; esto es debido a las aproximaciones y consideraciones que se hicieron en cada caso.

Orientación del Modelo de Working Model. La simulación teórica realizada a partir del modelo de Working Model muestra que la orientación o ángulo de pierna $\psi$ que alcanza la máquina de MPC en el movimiento de flexión-extensión tiene como valor máximo $135^{\circ}$ (figura 7).

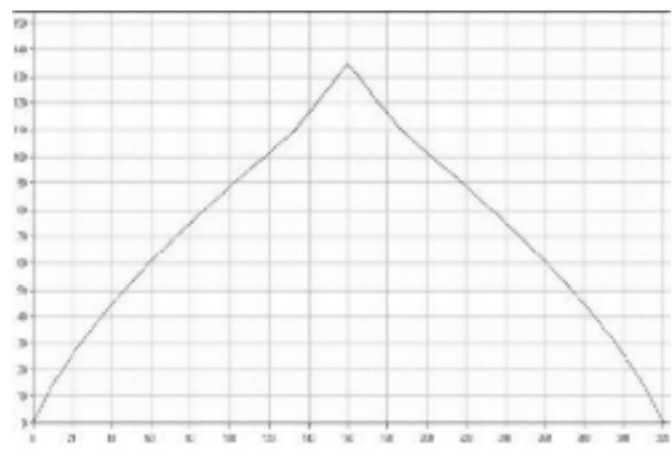

Figura 7: Orientación o Ángulo de Rodilla en función del Tiempo 


\subsection{Descripción de la Máquina de MPC}

La máquina de MPC se construyó con tubos de acero inoxidable ASTM 316. Se utilizaron bujes de bronce fosforado para el acople entre la tuerca y el tornillo de potencia y aluminio para las bases y la tuerca. Se utilizaron dos rodamientos autoalineantes de carga axial y radial.

La unidad consiste de una base, soporte para el pie, la pierna y el muslo, tornillo de potencia, eje de apoyo del tornillo de potencia, pasadores, motor de corriente continua, plantillas de felpa.

\subsection{Análisis de Esfuerzos}

Se toma la fuerza de flexión máxima $V$ en el centro del eje del tornillo de potencia para desarrollar los cálculos, la fuerza de empuje $Q$ y el torque $T$ como se ve en la figura 8.

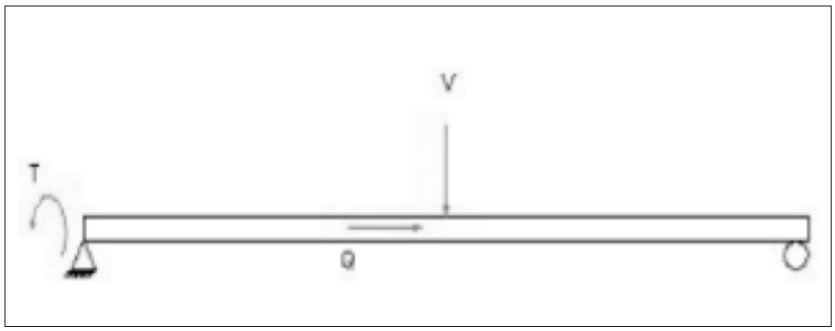

Figura 8: Estado de cargas del tornillo

Se toma el tornillo con un diámetro exterior $d_{e}=15,875 \mathrm{~mm}$, paso $3,275 \mathrm{~mm}$ y con condiciones de carga de flexión alternante, axiales medios y cortantes medios debido al comportamiento de las cargas, fuerza de flexión $V=100 N$, fuerza de empuje $Q=80 N$, coeficiente de fricción $\mu=0,35$, longitud del tornillo de 560 $\mathrm{mm}$. Con los valores máximos de las fuerzas de flexión, torsión y axial, se calculan los diferentes esfuerzos, con el círculo de Mohr [7] se obtienen los esfuerzos principales para los diferentes tipos de cargas. Utilizando la teoría de Misses-Hencky [7] o energía de distorsión se obtienen los esfuerzos equivalentes:

$$
\sigma^{*}=\left[\frac{\left(\sigma_{1}-\sigma_{2}\right)^{2}+\left(\sigma_{2}-\sigma_{3}\right)^{2}+\left(\sigma_{1}-\sigma_{3}\right)^{2}}{2}\right]^{1 / 2}
$$

Donde:

$\sigma *=$ Esfuerzo de Von Mises

$\sigma_{1}, \sigma_{2}, \sigma_{3}=$ Esfuerzos principales

Utilizando la teoría de Soderberg [6]:

$$
\frac{\sigma_{\mathrm{a}}}{S_{e}}+\frac{\sigma_{m}}{S_{y l}}=\frac{1}{F_{s}}
$$

$S_{e}=$ Limite de resistencia a la fatiga del elemento Mecánico

$\sigma_{\alpha}=$ Esfuerzos alternos

$\sigma_{m}=$ Esfuerzos medios

$S_{y l}=$ Resistencia a la cedencia

$F_{s}=$ Factor de seguridad

$$
\frac{59,50}{366,86}+\frac{1}{276}=\frac{1}{F_{s}}
$$

Se obtiene el factor de seguridad del tornillo de potencia que es igual a $F_{s}=6$

Para calcular la deflexión máxima $y_{\max }$ :

$$
y_{\max }=\frac{V L^{3}}{48 E I}
$$

Donde:

$L=$ Longitud entre los dos apoyos

$E=$ Módulo de elasticidad

$I=$ Momento de inercia

$$
y_{\max }=\frac{V L^{3}}{48 E I}=\frac{50(0,56)^{3}}{48\left(190 * 10^{6}\right)\left(2,485 * 10^{-9}\right)}=0.087 \mathrm{~mm}
$$

Lo que el diseño por rigidez y resistencia no tiene ningún problema, por lo tanto el diseño se encuentra en una área segura.

\subsection{Sistema de Control}

La figura 9 representa el diagrama general de bloques del controlador, acoplado con el módulo actuador [8], [9] y con el módulo comparador.

Módulo controlador. Compuesto de tres etapas separadas para establecer los valores deseados de:

-Rango de movimiento

-Posición inicial 
- Velocidad

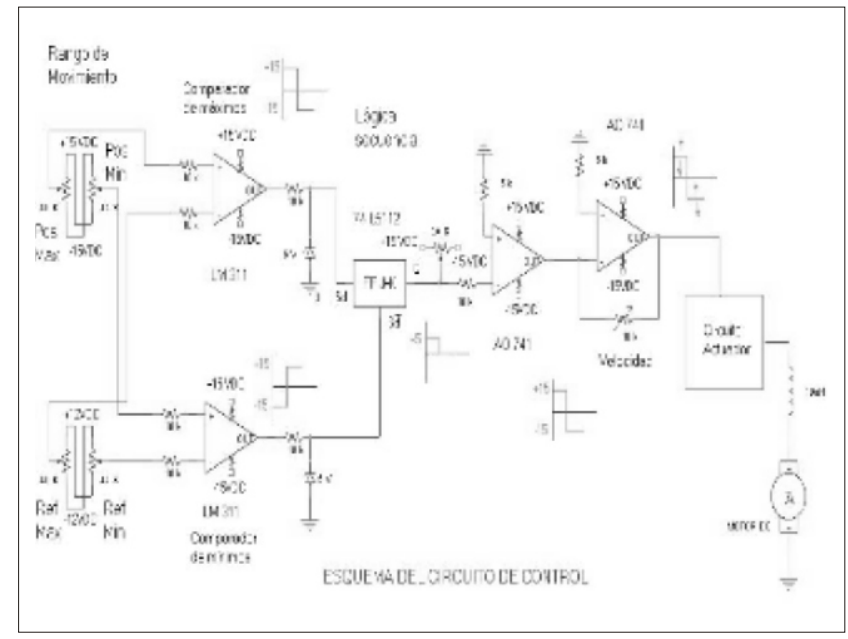

Figura 9: Esquema del Circuito de Control

Subsistema de control de rango de movimiento. Se prevee la posibilidad de obtener un ángulo de rotación de $0^{\circ}-135^{\circ}$. Se puede controlar a voluntad. Este dispositivo opera en lazo cerrado comparando las señales de posición actual máxima y mínima con las señales de referencia de posición también de máxima y mínima establecidas por el médico.

Subsistema de Posición Inicial. Proporciona la posibilidad de establecer el punto de inicio de carrera de desplazamiento.

Subsistema de Control de Velocidad. Las velocidades de desplazamiento del mecanismo de MPC, son relativamente bajas, del orden de 1 a 10 min. por ciclo completo $\left(0^{\circ}-90^{\circ}-0^{\circ}\right)$. Son establecidas por el médico de acuerdo a las características de cada caso en particular. Se diseñó un accionamiento de ganancia proporcional variable. Para garantizar el suministro de energía al sistema se diseña y construye una fuente de poder regulada dual.

Construcción del Módulo Actuador. Para el manejo de potencia se diseña y construye el módulo actuador compuesto por el amplificador operacional LM344 tipo industrial, dos transistores complementarios 2N3055 y Mj2955 para el manejo del circuito de potencia del motor (figura 10).

\section{PRUEBAS DE DESEMPEÑO DE LA MÁQUINA DE MPC}

Se evaluó el funcionamiento de la máquina de MPC durante los movimientos de flexión extensión. Se realizaron las siguientes pruebas:

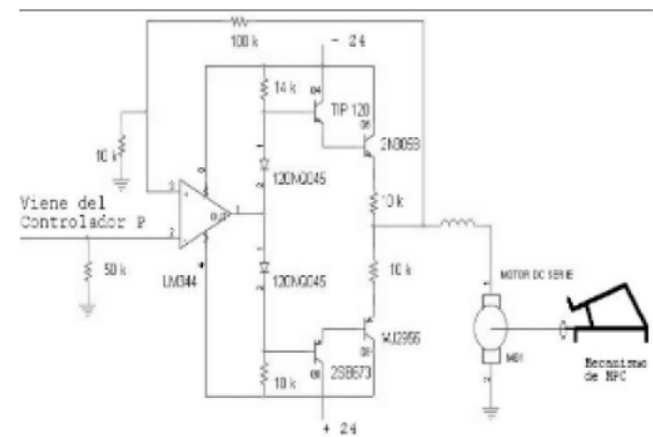

Figura 10: Circuito Electrónico del Módulo Actuador

\subsection{Sistema de Control}

El sistema de control automático de rango de movimiento, de velocidad y de posición inicial funciona de acuerdo a las expectativas especificadas en el diseño.

\subsection{Rango de Movimiento}

Se alcanza un rango de movimiento de $5^{\circ}$ a $132^{\circ}$, apropiado para la mayoría de las terapias medicas.

\subsection{Ruido Generado por la Máquina de MPC} La figura 11 muestra dicho comportamiento. La EPA (Environmental Protection Agency) ha determinado niveles de sonido menores a 45 $d B A$. El nivel de ruido [10] equivalente $L_{e q}$ si el intervalo de tiempo es corto ( $<1$ hora) se define como:

$$
L_{e q}=10 \log \left|\frac{1}{N} \sum_{i=1}^{N} 10^{\frac{L i}{10}}\right|
$$

Donde:

$L g=$ Logaritmo base 10

$L i=$ Mediciones de ruido

$N_{i}=$ Múmero de mediciones 
La máquina de MPC funciona con un nivel de ruido equivalente $L$ eq de 9,77 dBA, el cual se encuentra dentro de los valores aceptados por la EPA.

\subsection{Potencia del Motor}

La potencia mecánica fue calculada por medio de las simulaciones virtuales y analíticas, y medida experimentalmente (figura 12).

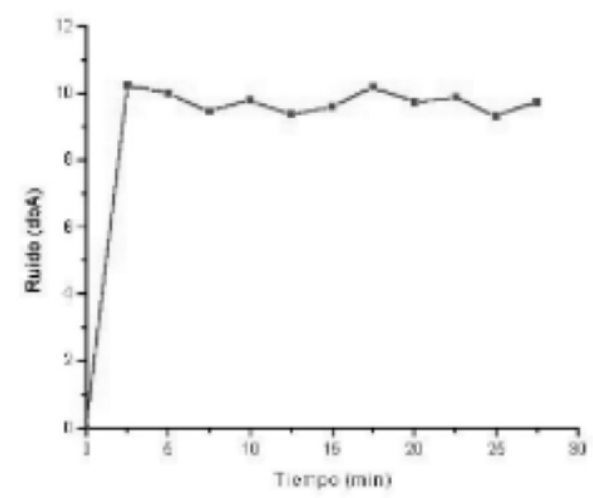

Figura 11: Ruido Generado por la Máquina de MPC

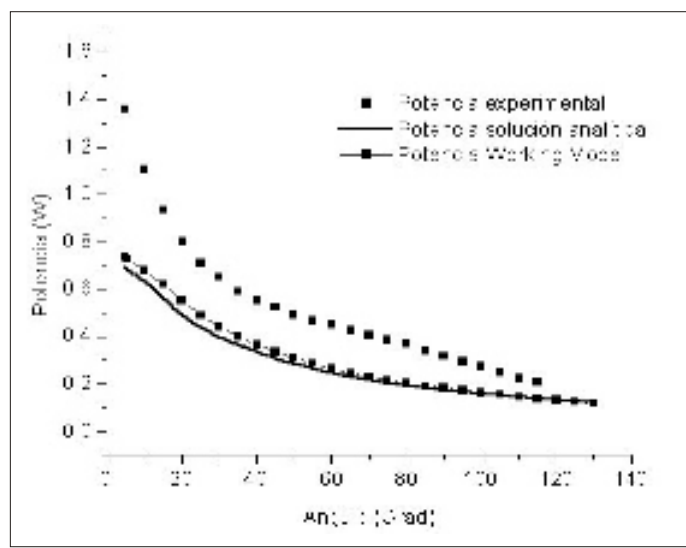

Figura 12: Potencia Mecánica o de Salida del Motor

\subsection{Rango de Velocidades}

Las velocidades alcanzables por el mecanismo de MPC son de 1 a 9 minutos por cada ciclo completo.

\section{CONCLUSIONES}

Se diseñó, construyó y se evaluó un prototipo de MPC para la terapia de rodilla con las siguientes características:

Rangos de movimientos ajustables

Flexión-extensión rodilla $5^{\circ}$ a $132^{\circ}$

Barras ecualizables

Fácil de operar

Alineación anatómica para el eje de movimiento

Para pierna izquierda y derecha

Velocidades variables

Para un amplio rango de personas (15 años a un adulto)

La máquina está diseñada para la articulación de rodilla y puede ser utilizada para la terapia de cadera, los movimientos que se desarrollan para la rodilla son los mismos que para la terapia de cadera, excepto en sus arcos de movilidad.

La máquina de MPC genera niveles de ruidos equivalente Leq de 9,77 dBA, lo cual está entre lo permitido por la Environmental Protection Agency.

Esta máquina es una solución en la búsqueda del mejoramiento de la calidad de vida de la población, debido a la disminución de costos tanto de fabricación y de alquiler, buscando siempre la masificación del tratamiento de lesiones.

\section{RECOMENDACIONES}

Para mejorar el diseño, podría pensarse en cambiar el sistema electromecánico (tornillo de potencia y motor eléctrico) por un nuevo sistema electromagnético, todo esto para evitar las pérdidas debidas a la fricción y a la cantidad de elementos.

Desarrollar sistemas de control automático que sean de tipo digital y a control remoto.

La máquina de MPC debe construirse con materiales que no generen infección a las heridas quirúrgicas. Deben utilizarse materiales no conductores o materiales aislantes.

Con la modernización del equipo se podrían 
realizar tratamientos muy complicados automáticamente a costos moderados, donde varias máquinas puedan ser manejadas desde un mando central computarizado con las respectivas estandarizaciones de los tratamientos, en la perspectiva de llegar aun control inteligente.

\section{REFERENCIAS}

[1.] McIMNES J. et all. A Controlled Evaluation of Continuous Passive Motion in Patients Undergoing Total Knee Arthroplast, Jama. Vol. 268, Mo.11, September 16, 1992.

[2.] ALFREDSOM H. Lorentzon R. Superior results with continuous passive motion compared to active motion after periosteal transplantation. A retrospective study of human patella cartilage defect treatment. , Vol 7, MO 4, 1999.

[3.] KAPAMDJI I. A. Cartillas de Fisiología Articular. Esquemas comentados por mecánica articular. Cuaderno II, La Rodilla. México: Masson, 1985.

[4.] CROMEY, John. Antropometría para Diseñadores.

[5.] WIMTER, David A. Biomechanics and Motor Control of Human Movement. Edition John Wiley \&Sons, Inc. Waterloo, Ontario, 1990.

[6.] HIROMICHI Fujie, GLEM A. Livesay, MASAHIRO Fujita and SAVIO L-Y. Woo. Forces and Moments in Six-DOF at the Human Knee Joint: Mathematical Description for Control. Journals of Biomechanics, Vol. 29, Mo. 12, Pp. 1577-1585, 1996.

[7.] SHIGLEY Joseph Edward y MISCHKE Charles. Diseño en Ingeniería Mecánica. España: Editorial McGraw-Hill, 1990.
[8.] OGATA Katsuhiko. Ingeniería de Control Moderna. México. Prenticce Hall. Tercera Edición, 1998.

[9.] KUO Benjamín. Sistemas de Control Automático. México: Prentice Hall Hispanoamericana S.A., 1996.

[10.] WILSOM Charles. Computer Integrated Machine Design. U.S.A: Editorial Prentice Hall, 1997.

[11.] WebsteR, John. Medical Instrumentation: Application and Design. Second edition. U:S:A. Edition for Houghton Mifflin Company, 1992. 